\title{
Clustering Algorithm of Wireless Sensor Networks Based on Residual Energy
}

\author{
Ma Changlin ${ }^{1, a}$, Ruan Yuan ${ }^{1, b}$ \\ ${ }^{1}$ School of Computer, Central China Normal University, Wuhan 430079, China \\ aclma@mail.ccnu.edu.cn, r ruanyuan2008@126.com
}

Keywords: wireless sensor networks, residual energy, clustering algorithm, NS2 simulation

\begin{abstract}
In order to improve the lifetime and throughput of wireless sensor networks under the limited power, an improved clustering algorithm is proposed in this paper on the basis of LEACH protocol. The energy factor is considered in this algorithm. The residual energy of all sensor nodes is referred to select cluster-heads of wireless sensor networks. The new clustering algorithm effectively improves the energy efficiency, throughput and lifetime of wireless sensor networks. The results are proved by simulations.
\end{abstract}

\section{Introduction}

The rapid development of the electronic components has promoted the low power, low-cost and multi-functional sensor nodes to appear. The sensor nodes can not only sense information but also compute and communicate. In addition, they gather to form wireless sensor networks (WSNs) $[1,2]$. WSNs are widely applied in military detection, environmental monitoring, industrial control, intelligent buildings, transportation and other commercial areas [3, 4]. In general, sensor nodes are scattered in the sensing field. In addition to sensing data, the nodes can process the acquired information and transmit messages to the distant base station (BS). Finally they transmit the collected information to the users. The architecture of the WSNs is depicted in Fig. 1 [5].

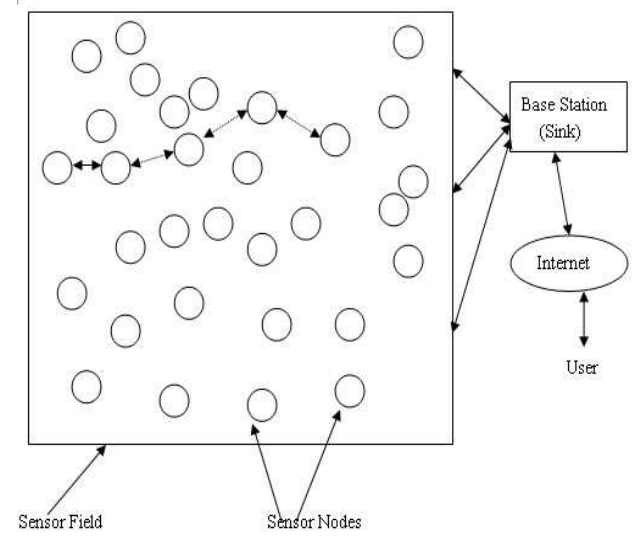

Fig. 1. The architecture of WSNs

There are two categories for the existing data-gathering protocols: hierarchy (cluster-based) protocols and non-hierarchy protocols. The non-hierarchy protocols [6, 7] were used by early researchers, i.e., each node directly sends data packets to the BS. The shortages of the non-hierarchy protocols are that the nodes spend a lot of energy and the lifetime of network is reduced during data transmission. Besides, the same information may be repeatedly sensed by nodes nearby. It generates redundant data received by BS and increases the load of the system. Therefore, hierarchy routing protocols have been proposed by some researchers. By applying these hierarchy routing protocols, some relevant nodes form a cluster in the network. Compared with non-hierarchy routing protocols, clustering topology is easily managed and has good scalability. Since the member nodes have simple function, they do not need to maintain complex routing information. Thus the lifetime and throughput 
rate of WSNs are prolonged. In WSNs, one of the typical hierarchical routing protocols is LEACH protocol. The algorithm randomly selects cluster-heads according to a threshold value, which makes all the sensor nodes have opportunity to be cluster-heads. However, this kind of random selection may cause the instability of system, because the residual energy of WSNs is not considered in the algorithm. The system consumes excessive energy, increases the load of the system and decreases the lifetime of the network.

Considering the above problems, an improved clustering algorithm is presented in this paper. The energy factor is considered in the algorithm. The cluster-heads are selected based on the residual energy. Compared with LEACH, the new algorithm improves the lifetime, the throughput rate and the energy efficiency.

\section{Related Work}

LEACH algorithm was designed in [1]. The algorithm was divided into two phases: the cluster-head election phase and the cluster formation phase.

(1) Cluster-head election

LEACH algorithm used the following threshold to be the probability of being a cluster-head for each sensor node:

$$
T(n)= \begin{cases}\frac{k}{N-k^{*}\left(r \bmod \frac{N}{k}\right)} & \text { if } n \in G \\ 0 & \text { otherwise }\end{cases}
$$

where $N$ is the number of the nodes, $k$ is the expected number of cluster-heads, $r$ is the number of rounds that have passed, $k^{*}\left(r \bmod \frac{N}{k}\right)$ is the number of the nodes that have been elected as cluster-heads after $r$ rounds, and $G$ is the set of sensor nodes which have not been selected as cluster-heads yet. Firstly, each sensor node generates a random number between 0 and 1 . Then according to Eq. (1), the node is selected as a cluster-head if its random number is smaller than the threshold.

(2) Cluster formation

By the clustering algorithm, these cluster-heads send message to other nodes in the network through broadcast. Once common nodes have received the message from cluster-heads, it selects to join in the cluster nearby which consumes the least energy.

\section{Clustering Algorithm Based On Residual Energy}

According to section 2, in the cluster-head election phase of LEACH, Eq. (1) does not consider the effect of energy. This algorithm selects cluster-heads randomly, which causes instability of the system. Therefore, it leads to the following disadvantages: (1) Maybe there is no cluster-head in some rounds. Under such circumstances, the nodes will send the data directly to the BS. Now the routing protocol in the network is the same as non-hierarchy protocols which may consume a lot of energy and decrease the lifetime. (2) Some cluster-heads get together. It increases the distance which some nodes transmit the data to the BS. So it increases the load of the system and affects the lifetime of the network. (3) Once the nodes which have low residual energy are selected as cluster-heads, they will soon use up their energy so that the network will be locally or totally paralyzed.

Considering the above disadvantages, an improved clustering algorithm is presented in this paper. The energy factor is considered in the algorithm. The cluster-heads are selected based on the residual energy.

In WSNs, each sensor node has three states: sleep, idle, active. When the node is in the sleep state, the radio module and the processor module are shut down while the sensor module is active. In [1], $\mathrm{P}_{\text {sleep }} \mathrm{W}$ denotes the energy consumption of the node during sleep state. All modules are open and 
ready during the idle state. At the moment, there is no data processed or signals sent by the node. $\mathrm{P}_{\text {idle }} \mathrm{W}$ denotes the energy consumption of the node during idle state. During the active state, the node receives, transmits and fuses data. Fig. 2 shows the radio energy model which the nodes receive and send data [1].

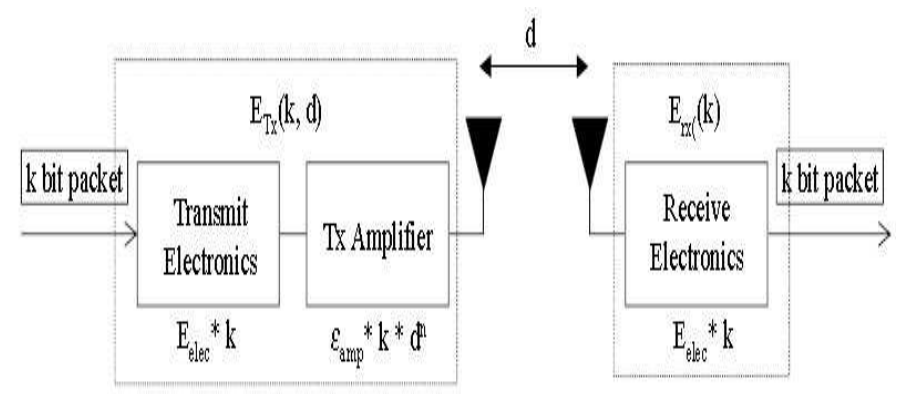

Fig. 2. Radio energy consumption model

In [1], the equation of energy model is as follows:

Eq. (2) represents the transmitting energy of a node. Eq. (3) is the receiving energy of a node.

$$
\begin{gathered}
E_{T x}(l, d)= \begin{cases}l E_{\text {elec }}+l \varepsilon_{\text {friss-amp }} d^{2} & d<d_{\text {crossover }} \\
l E_{\text {elec }}+l \varepsilon_{\text {two-ray-amp }} & d \geq d_{\text {crossover }}\end{cases} \\
E_{R x}(l)=l E_{\text {elec }}
\end{gathered}
$$

where $l$ is the length of data, $d$ is the distance of two nodes, $d_{\text {crossover }}$ is the critical distance of two nodes through which the system judge to use corresponding power consumption model, $E_{\text {elec }}$ is the radio energy consumed by transmitting data per bit, $\varepsilon_{\text {friss-amp }}$ and $\varepsilon_{\text {two-ray-amp }}$ are parameters of the transmitter to amplify the signals, $E_{D A}$ is the energy consumed by cluster-head to fuse the data per bit, which is considered in the following simulations.

After the initial energy subtracts the total energy consumed during sleep, idle and active state, we get the result as the residual energy $E_{i}(t)$. The clustering algorithm in this paper is based on residual energy (CARE). The main idea of CARE is as follows:

Given the equal initial energy, the BS considers the effect of the residual energy when selecting the cluster-heads. Improving Eq. (1), we get Eq. (4).

$$
T(n)=\left\{\begin{array}{l}
\frac{k}{N-k *\left(r \bmod \frac{N}{k}\right)} * E_{i}(t) \text { if } n \in G \\
0 \quad \text { otherwise }
\end{array}\right.
$$

where $E_{i}(t)$ is the residual energy. Hence, if one node has more residual energy, it will generate a larger threshold. There is no doubt that it has more possibility to be a cluster-head. It can avoid using up the energy of some nodes whose energy is low. Therefore, our new algorithm can enhance the performance of the network.

\section{Simulations}

In this paper, we use NS2 simulation tools [8-10]. The clustering algorithm CARE is simulated. The simulation environment is set up as follows. The sensor network area considered in the simulation is $200 \mathrm{~m} \times 200 \mathrm{~m}$. There are 100 sensor nodes and one base station. The expected cluster-heads are five. The simulation parameters are shown in Table 1: 
Table 1 Parameters used in the simulation

\begin{tabular}{lc}
\hline Items & Values \\
Network area & $200 \mathrm{~m} \times 200 \mathrm{~m}$ \\
Initial energy & $2 \mathrm{~J}$ \\
Simulation time & $500 \mathrm{~s}$ \\
Base Station location & $(50,75)$ \\
\hline
\end{tabular}

In this experiment, the throughput, energy consumption and lifetime of the network are simulated. We compare the results obtained by CARE with those of using LEACH protocol under the same circumstances. As shown in Fig.3, the throughput of CARE is higher than LEACH. It is approximately increased by $25 \%$. Fig. 4 shows the new algorithm has lower energy consumption than LEACH algorithm, which is approximately decreased by $31 \%$. In WSNs, the researchers focus on the lifetime. Fig.5 shows the death rate of the nodes through which we can conclude that the new algorithm has longer lifetime than LEACH. The lifetime is approximately increased by $11 \%$.

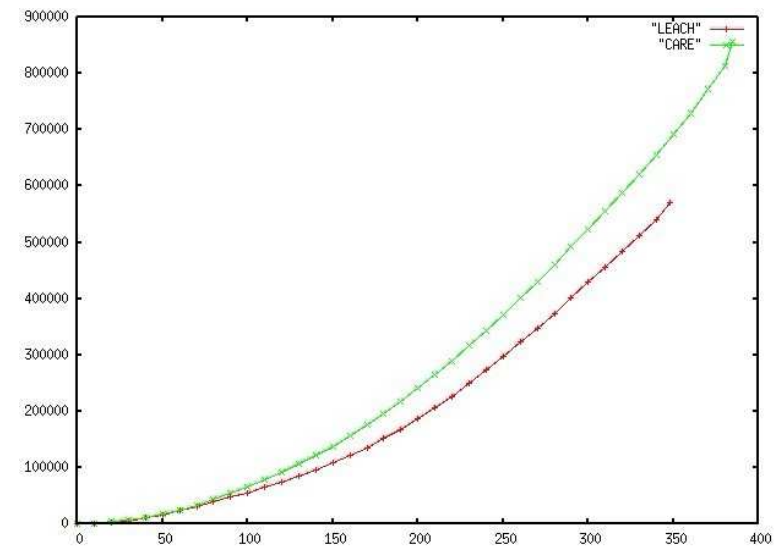

Fig.3. Comparison of the throughput

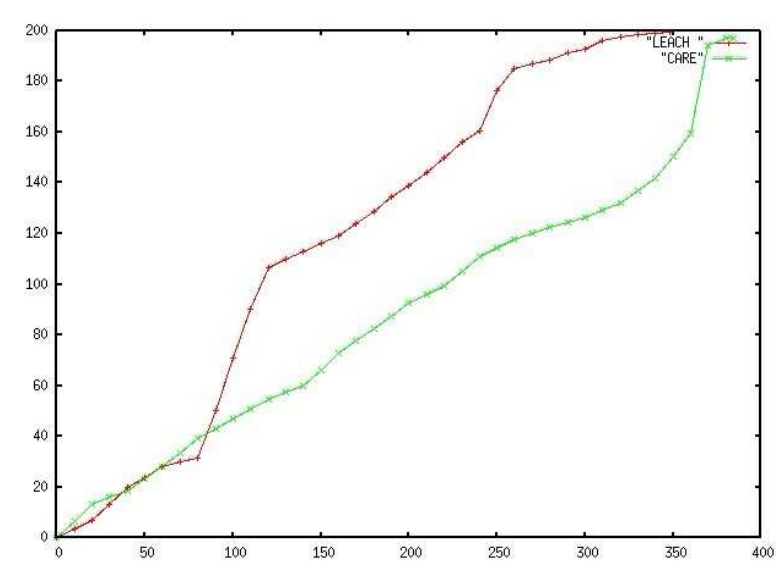

Fig.4. Comparison of the energy consumption

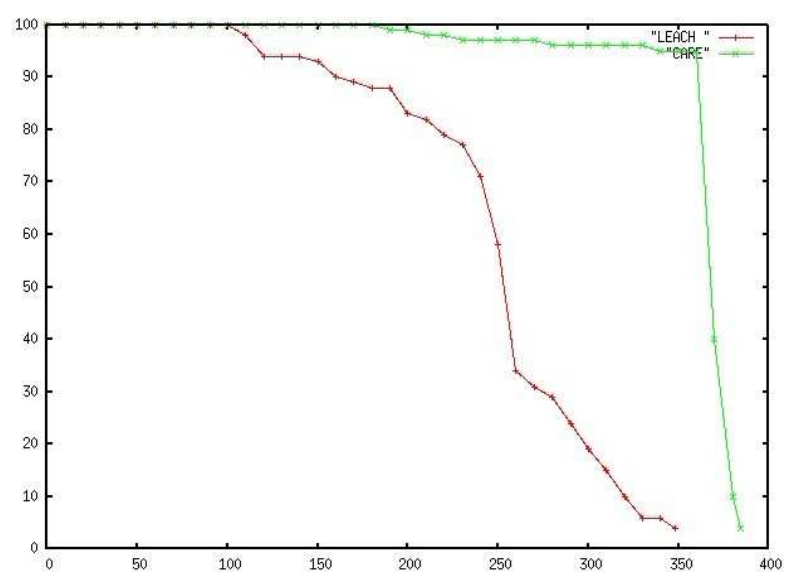

Fig.5. Comparison of the lifetime

\section{Conclusions}

In order to improve the lifetime and throughput rate of WSNs, an improved clustering algorithm is proposed in this paper. The residual energy of sensor nodes is considered to select cluster-heads of WSNs. The result is verified by simulations. Compared with LEACH, the algorithm improves the lifetime, the throughput rate and the energy efficiency. 


\section{References}

[1] Wend Beth Heinzelman, Application-Specific Protocol Architectures for Wireless Networks. Massachusetts: Department of Electrical Engineering and Computer Science, Massachusets Institute of Technology, 2000.

[2] John A. Stankovic, Wireless Sensor Networks. Charlottesville: Department of Computer Science, University of Virginia,2006.

[3] K. K. Khedo, R. Perseedoss and A. Mungur, "Wireless Sensor Network Air Pollution Monitoring System", International Journal of Wireless \& Mobile Networks(IJWMN),Vol.2(2), 2010.

[4] Carlos F. Garca, "Wireless Sensor Networks and Applications: a Survey", International Journal of Computer Science and Network Security(IJCSNS), Vol.7(3), 2007.

[5] Chuan-Ming Liu, Chuan-Hsiu Lee and Li-Chun Wang, "Distributed clustering algorithms for data-gathering in wireless mobile sensor networks," Journal of Parallel and Distributed Computing,pp:1187-1200, 2007.

[6] Shio Kumar Singh, M P Singh and D K Singh, "Routing Protocols in Wireless Sensor Networks-A Survey, International Journal of Computer Science \& Engineering Survey(IJCESE), Vol.1(2), 2010.

[7] L. J. G. Villaba, A. L. S. Orozco, A. T. Cabrera etc, "Routing Protocols in Wireless Sensor Networks", Sensors, 2009, 9, 8399-8421, 26 October 2009.

[8] Zhiheng Ke, Rongxiang Cheng, Dejun Deng. NS2 Simulation Experiment. Beijing: Electronic Industry Press, 2008.

[9] Luping Fang, Shihua Liu, Pan Chen. Basis and Applications of NS-2 Network Simulation. Beijing: National Defense Industry Press, 2008.

[10]Bin Yu, Bin Sun, Nuan Wen, NS2 and Network Simulation. Beijing: The People's Posts and Telecommunications Press, 2007. 